\title{
Effect of Proline Pretreatment on Grapevine Shoot-Tip Response to a Droplet-Vitrification Protocol
}

\author{
Zvjezdana Marković $^{1,2,3}$, Philippe Chatelet ${ }^{2,3}$, André Peyrière ${ }^{2}$, Darko Preiner ${ }^{1}$, \\ Isabelle Engelmann-Sylvestre ${ }^{3}$, Jasminka Karoglan Kontić ${ }^{1}$, Florent Engelmann ${ }^{3}$ \\ ${ }^{1}$ Faculty of Agriculture, University of Zagreb, Zagreb, Croatia; ${ }^{2}$ INRA, UMR AGAP, Equipe DAVEM, Montpellier, France; ${ }^{3}$ IRD, \\ UMR DIADE, Montpellier, France. \\ Email: florent.engelmann@ird.fr
}

Received October $1^{\text {st }}, 2013$; revised November $2^{\text {nd }}, 2013$; accepted November $15^{\text {th }}, 2013$

Copyright (C) 2013 Zvjezdana Marković et al. This is an open access article distributed under the Creative Commons Attribution License, which permits unrestricted use, distribution, and reproduction in any medium, provided the original work is properly cited.

\begin{abstract}
Proline has been shown to accumulate in plants in response to biotic and abiotic stresses. Exogenous proline has thus been used for improving some plant cryopreservation protocols. Further enhancement of cryopreservation efficiency for in vitro grapevines could be expected if stresses linked to cryopreservation procedures could be reduced. We therefore studied the possible beneficial effect of proline in grapevine cryopreservation. Single-node explants from in vitro grown grapevine plantlets (Vitis vinifera L. cv Portan) were cultured on shooting media (half-strength MS $+1 \mu \mathrm{M} \mathrm{BAP}$ ) containing no proline (control) or 50, 500, or $2000 \mu \mathrm{M}$ filter-sterilized L-proline. Shoot tips excised from these microshoots were subjected to a PVS2-based droplet-vitrification procedure. Control and rewarmed explants were grown on a recovery medium containing $1 \mu \mathrm{M}$ BAP. Shoot development on control medium and lower proline concentrations did not notably differ whereas the highest concentration of proline inhibited shoot development. Carry-over effects were observed since lower survival and regrowth were obtained both for non-frozen or LN-treated explants excised from micro-shoots obtained on the $2000 \mu \mathrm{M}$ proline medium. No significant differences in survival and regrowth were observed for non-frozen explants subjected to pretreatment without LN exposure. A slightly enhancing effect (although non-significant) on post-cryopreservation survival was observed for explants derived from shoots developed on 50 or $500 \mu \mathrm{M}$ proline, but no significant improvement of regrowth percentage was observed for these two conditions. Although a slight increase in survival could be observed, no significant beneficial effect of proline pretreatment on postcryoconservation regrowth could be evidenced in our conditions. However, the 2-week period before explant excision could have allowed at least partial metabolism and catabolism of exogenous proline; the results observed could thus have been the consequence of complex interactions. Shorter proline treatments applied closer to the actual LN exposure step might produce different results and allow for clearer interpretation.
\end{abstract}

Keywords: Vitis vinifera L.; Cryopreservation; In Vitro Culture; Stress Alleviation; Amino Acid; Medium Supplementation

\section{Introduction}

Proline has been shown to accumulate in plant cells and organs in response to biotic (plant-pathogen interaction) and abiotic (water limitation, redox potential modification, freezing, etc.) stresses, even though this amino acid seems to accumulate in highest concentrations in the event of a drought stress [1]. These stress-mitigating properties have prompted the inclusion of exogenous proline in some plant cryopreservation protocols in order to improve explant survival and regrowth [2]. Although its modes of action are not fully known, proline could likely act protectively during the dehydration steps included in all cryopreservation protocols. Indeed, dehydration is known to evoke the formation of reactive oxygen species [3] and exogenous proline has recently been shown to alleviate $\mathrm{H}_{2} \mathrm{O}_{2}$-mediated oxidative stress in grapevine leaves [4].

Thus, while satisfactory efficiency of cryopreservation is documented for in vitro grapevines [5], further improvement could be expected if stresses linked to cryopreservation procedures (such as oxidative stress) could be reduced. To our knowledge the possible beneficial 
effect of proline in grapevine cryopreservation has not been tested yet and we therefore considered it worthwhile to investigate its influence in a vitrification-based protocol.

\section{Materials and Methods}

Single-node explants isolated from in vitro-grown grapevine plantlets (Vitis vinifera L. cv Portan) were cultured for 2 weeks on shooting media (half-strength MS-based medium plus $1 \mu \mathrm{M}$ BAP) containing no proline (control) or 50,500 , or $2000 \mu \mathrm{M}$ filter-sterilized L-proline.

After 2 weeks of culture of single-node explants (at least 37 explants per experimental condition) on the different shooting media tested, the number of shoot-developing nodes was counted and individual shoot length and number of newly-developed nodes were recorded. Shoot tips excised from micro-shoots obtained on the different shooting media were subjected to a PVS2-based droplet-vitrification protocol [6] and plunged into liquid nitrogen (LN) for at least $1 \mathrm{~h}$. Control explants (treated with loading, PVS2, and unloading solutions, but not exposed to $\mathrm{LN}$ ) and LN-treated, rewarmed explants were grown on the same recovery medium containing $1 \mu \mathrm{M}$ BAP. Thirteen to eighteen explants were used per condition.

\section{Results and Discussion}

\subsection{Effect of Proline on Shoot Development and Growth Parameters}

Shoots developed on the different shooting media did not demonstrate notable differences in aspect (Figure 1) although leaves produced on media with the higher two

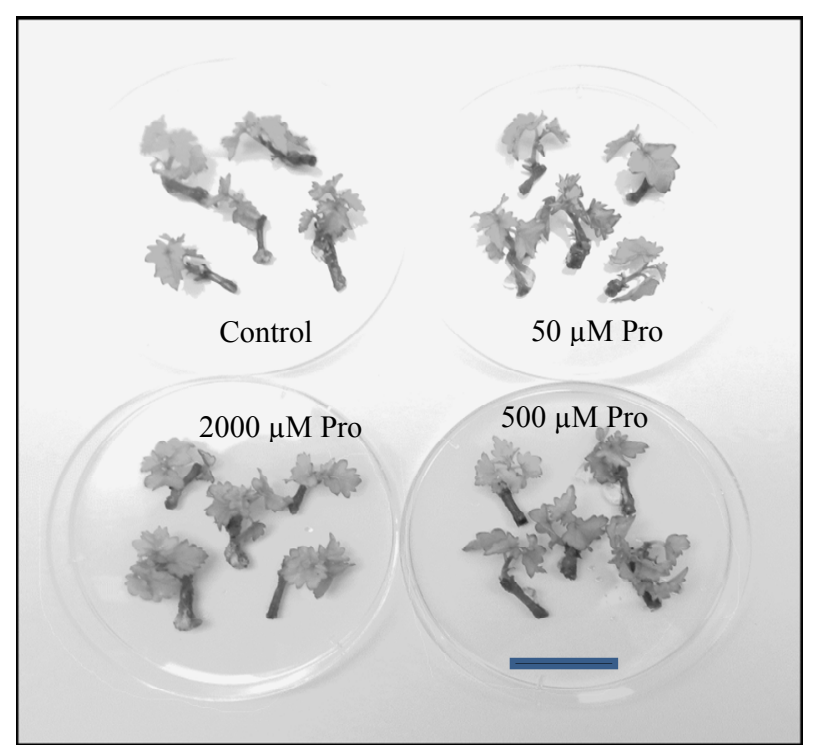

Figure 1. Representative samples of shoots developed after 2 weeks on different shooting media. $B a r=2 \mathrm{~cm}$. proline concentrations displayed a slightly lighter green; similarly, a comparable number of shoots and nodes were produced over the range of media, although a slightly lower number of nodes were formed on the medium containing the highest proline concentration (Figure 2).

Shoots arising from the control medium and media with the lower two proline concentrations did not notably differ in their development with similar shoot length measured, whereas the highest proline concentration inhibited shoot development (Figure 3) with a significant global difference between treatments. As no other pairwise comparisons were significant, this could be explained by a slightly enhancing effect of proline used at $500 \mu \mathrm{M}$ and a slightly depressive effect of the $2000 \mu \mathrm{M}$ treatment, both not large enough to differ from the control but statistically different when compared. Thus, in our conditions, proline did not markedly alter shoot development although some toxicity could be suspected at high concentrations. Indeed, excess proline has been shown to negatively affect chlorophyll-binding proteins [7], which could account for the loss of coloration observed at the highest two proline concentrations. In addition, both the number of nodes formed and micro-shoot length mean value were lower (but not significantly)

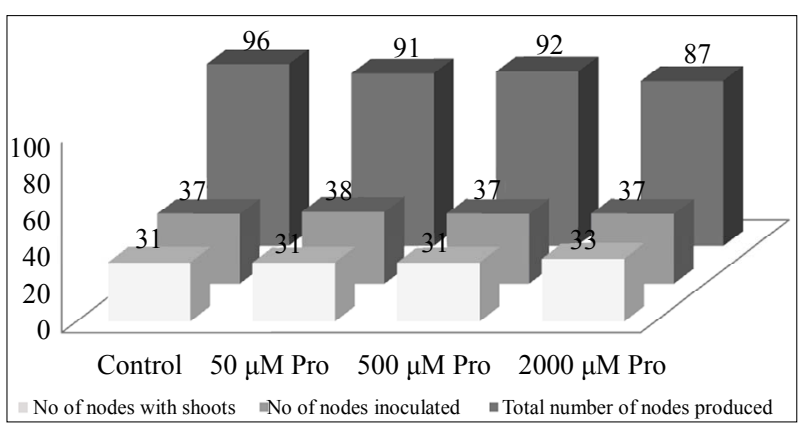

Figure 2. Number of shoots developed and total number of nodes formed 2 weeks after inoculation on the different shooting media.

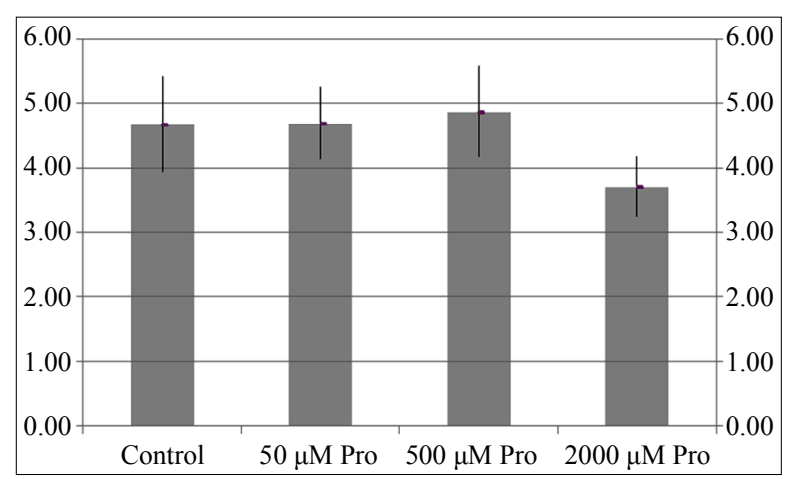

Figure 3. Mean shoot length $(\mathrm{mm})$ obtained on the different shooting media with $\alpha=5 \%$ confidence intervals. Mean values differing by one letter are significantly different (Student's t-test, $\alpha=\mathbf{5 \%}$ ). 
Table 1. Percentage of survival and regrowth (observed after 2 and 8 weeks, respectively) of control ( $-\mathrm{LN}$ ) and cryopreserved $(+\mathrm{LN})$ shoot tips excised from different shooting media and subjected to a PVS2-based droplet-vitrification protocol.

\begin{tabular}{ccccc}
\hline Shooting medium & Survival & & Regrowth \\
\hline & $-\mathrm{LN}$ & $+\mathrm{LN}$ & $-\mathrm{LN}$ & $+\mathrm{LN}$ \\
0 Pro & 52.9 & 27.8 & 41.4 & 50.0 \\
$50 \mu \mathrm{M}$ Pro & 57.1 & 38.8 & 61.5 & 33.3 \\
$500 \mu \mathrm{M}$ Pro & 61.5 & 40.0 & 28.5 & 30.0 \\
$2000 \mu \mathrm{M}$ Pro & 42.8 & 23.0 & $\mathrm{~ns}$ & 15.4 \\
& $\mathrm{~ns}^{*}$ & $\mathrm{~ns}$ & $\mathrm{~ns}$ \\
\hline
\end{tabular}

when explants were grown at the highest proline concentration.

\subsection{Effect of Proline on Post-Cryopreservation Survival and Regrowth}

After explant pretreatment with loading/PVS2 solutions with or without LN exposure, shoot tip survival and regrowth were recorded after 2 and 8 weeks (Table 1). Carry-over effects for the $2000 \mu \mathrm{M}$ proline medium were observed since lower survival and regrowth were obtained both for LN-treated or non-treated explants excised from micro-shoots obtained on this medium. No significant differences in survival and regrowth were observed for explants subjected to pretreatment phases without LN exposure. A slightly enhancing effect (although non-significant) on post-cryopreservation survival was observed for explants derived from shoots developed on 50 or $500 \mu \mathrm{M}$ proline, but no significant improvement of regrowth was observed for these two conditions.

Increasing explant number in repeat experiments would clearly improve statistical significance. However, the 2-week period before explant excision could have allowed at least partial catabolism of exogenous proline back to glutamate [8]. Exposure to exogenous proline at higher $(45 \mathrm{mM})$ concentration has also been shown to modify the endogenous amino acid pool [9]. We therefore cannot rule out the fact that interpretation of the results observed in our conditions could have been made difficult as a result of complex interactions. Shorter exposure to proline can then be envisaged in order to reduce proline catabolism and better evidence its proper effect. The actual rate of proline uptake by in vitro grapevine explants is not known, but endogenous proline content of Arabidopsis seedlings was increased almost 100-fold within $24 \mathrm{~h}$ when they were treated with $45 \mathrm{mM}$ proline [9] and a 6-h treatment with $20 \mathrm{mM}$ proline was sufficient to effectively reduce oxidative stress in grapevine leaves excised from in vitro plants [4]. It is therefore likely that shorter proline treatments applied just before
LNexposure (i.e. in the 24-h period after explant excision and before explant pretreatment with vitrification solutions) could evoke a clearer response as to the possible protective effect of proline during grapevine in vitro shoot tip cryopreservation.

\section{Conclusion}

Over the range of concentrations tested, proline did not strongly alter shoot development. Detrimental carry-over effects of proline at $2000 \mu \mathrm{M}$ could be suspected as shoot tips regrowth after vitrification treatments and LN exposure was negatively affected. After LN exposure, although a slight increase in survival could be observed at the lowest concentrations tested, no significant effect of proline pretreatment on post-cryopreservation regrowth could be evidenced in our conditions. However, repeating the experiment with shorter durations of exposure to proline would likely allow clearer interpretation by limiting the effects of proline metabolism and likely interactions resulting therefrom.

\section{Acknowledgements}

This work has been supported by grants from the French Ministry of Foreign Affairs (Z. Marković) and from ARCAD, a flagship programme of Agropolis Fondation (Montpellier, France) (I. Engelmann-Sylvestre).

\section{REFERENCES}

[1] P. E. Verslues and S. Sharma, "Proline Metabolism and Its Implications for Plant-Environment Interaction," Arabidopsis Book, 2010. http://dx.doi.org/10.1199/tab.0140

[2] D. J. Burritt, "Proline and the Cryopreservation of Plant Tissues: Functions and Practical Applications," In: I. Katkov, Ed., Current Frontiers in Cryopreservation, InTech Open Access Publisher, 2012, pp. 415-426.

http://www.intechopen.com/books/current-frontiers-in-cr yopreservation http://dx.doi.org/10.5772/35919 
[3] N. Smirnoff, "The Role of Active Oxygen in the Response of Plants to Water Deficit and Desiccation," New Phytologist, Vol. 125, No. 1, 1993, pp. 27-58. http://dx.doi.org/10.1111/j.1469-8137.1993.tb03863.x

[4] M. Ozden, U. Demirel and A. Kahraman, "Effects of Proline on Antioxidant System in Leaves of Grapevine (Vitis vinifera L.) Exposed to Oxidative Stress by $\mathrm{H}_{2} \mathrm{O}_{2}$," Scientia Horticulturae, Vol. 119, No. 2, 2009, pp. 163168. http://dx.doi.org/10.1016/j.scienta.2008.07.031

[5] C. Benelli, A. De Carlo and F. Engelmann, "Recent Advances in the Cryopreservation of Shoot-Derived Germplasm of Economically Important Fruit Trees of Actinidia, Diospyros, Malus, Olea, Prunus, Pyrus and Vitis," Biotechnology Advances, Vol. 31, No. 2, 2012, pp. 175-185. http://dx.doi.org/10.1016/j.biotechadv.2012.09.004

[6] Z. Marković, P. Chatelet, I. Sylvestre, J. Karoglan Kontić, and F. Engelmann, "Cryopreservation of Grapevine (Vitis vinifera L.) in Vitro Shoot Tips," Central European Jour- nal of Biology, Vol. 8, No. 10, 2013, pp. 993-1000. http://dx.doi.org/10.2478/s11535-013-0223-8

[7] T. Nanjo, M. Fujita, M. Seki, T. Kato, S. Tabata and K. Shinozaki, "Toxicity of Free Proline Revealed in an Arabidopsis T-DNA-Tagged Mutant Deficient in Proline Dehydrogenase," Plant and Cell Physiology, Vol. 44, No. 5, 2003, pp. 541-548. http://dx.doi.org/10.1093/pcp/pcg066

[8] L. Szabados and A. Savouré, "Proline: A Multifunctional Amino Acid," Trends in Plant Science, Vol. 15, No. 2, 2010, pp. 89-97.

http://dx.doi.org/10.1016/j.tplants.2009.11.009

[9] J. G. Chen, Y. Q. Zhang, C. P. Wang, W. T. Lü, J. B. Jin and X. J. Hua, "Proline Induces Calcium-Mediated Oxidative Burst and Salicylic Acid Signaling," Amino Acids, Vol. 40, No. 5, 2012, pp. 1473-1484. http://dx.doi.org/10.1007/s00726-010-0757-2 